\title{
Alcohol Consumption as a Risk Factor for Esophageal Adenocarcinoma in North China
}

\author{
Jun Chen, ${ }^{1}$ Nan Zhang, ${ }^{1}$ Yiwei Ling, ${ }^{1}$ Toshifumi Wakai, ${ }^{2}$ Yutong He, ${ }^{3}$ Lizhen Wei, ${ }^{3}$ \\ Shijie Wang ${ }^{3}$ and Kouhei Akazawa ${ }^{1}$

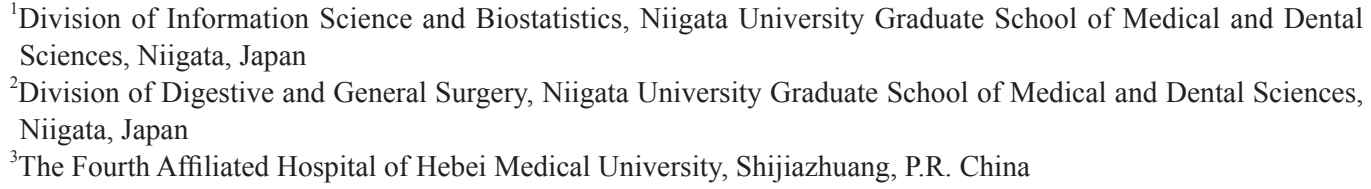

The incidence of esophageal adenocarcinoma has been rising in many countries, while esophageal squamous cell carcinoma has remained stable or even declined in the same populations over the identical periods. These differences in trends indicate that these cancer subtypes may have a different etiology, which may be caused by lifestyle factors such as alcohol consumption and cigarette smoking. Therefore, a matched case-control study to clarify the risk factors of alcohol and tobacco intake on the development of esophageal adenocarcinoma was collected in Hebei Province of China. The life expectancy of the study area was around 70 years old. In the present study, 98 patients younger than 65 years who were diagnosed with esophageal adenocarcinoma and had initial surgeries (cases) were matched with 294 healthy adults (controls) at a ratio of $1: 3$ according to sex and age. We found the proportions of drinkers and smokers among cases were $48.0 \%$ and $60.2 \%$, respectively, versus $21.2 \%$ and $43.5 \%$ among controls. Univariate conditional logistic regression analyses revealed that the odds ratios (ORs) showed a nearly monotonic increase for the duration of alcohol consumption and duration of tobacco smoking. Multivariate conditional logistic regression analysis indicated that only alcohol consumption was a significant risk factor for esophageal adenocarcinoma. Additional analysis of the combination of amount and duration of alcohol consumption indicated that heavy drinkers ( $>30 \mathrm{ml} /$ day) had significantly higher ORs, irrespective of the duration of alcohol consumption. In conclusion, heavy alcohol consumption increases the risk for esophageal adenocarcinoma independent of the duration of such consumption.

Keywords: alcohol consumption; tobacco smoking; esophageal adenocarcinoma; interaction between amount and duration; Chinese

Tohoku J. Exp. Med., 2011, 224 (1), 21-27. C 2011 Tohoku University Medical Press

The incidence of esophageal adenocarcinoma has been rising in many countries. In some populations this increase has been faster than that for any other major cancers. In contrast, the incidence of esophageal squamous cell carcinoma has remained stable or even declined in the same populations over the identical time periods (Blot et al. 1991; Pera et al. 1993; Chen et al. 1995; Devesa et al. 1998; Wei and Shaheen 2003). In the United States, the incidence of esophageal adenocarcinoma rose approximately six-fold, between 1975 to 2000 (Pohl and Welch 2005; Brown et al. 2008). Incidence has also been increasing in other Western countries, including the Netherlands and the United Kingdom (Botterweck et al. 2000; Bollschweiler et al. 2001; Crane et al. 2007). In China, economic conditions and the living environment have considerably improved over the past several decades. Nevertheless, studies have shown an increase in esophageal adenocarcinoma in both municipal and rural regions during this period (Fan et al. 2008).

The risk factors for squamous cell carcinoma have been well studied, low income, smoking, high alcohol intake, and low intake of raw fruits and vegetables account for a high proportion of new cases of esophageal squamous cell carcinoma (Brown et al. 2001; Engel et al. 2003; Freedman et al. 2007). However, factors underlying the rising incidence of esophageal adenocarcinoma are unclear. Alcohol consumption and tobacco smoking are established risk factors for esophageal cancer. However, they may be differentially associated with esophageal squamous cell carcinoma and esophageal adenocarcinoma. Therefore, it is

Received January 4, 2011; revision accepted for publication April 4, 2011. doi: 10.1620/tjem.224.21

Correspondence: Jun Chen, Department of Medical Informatics, Niigata University Graduate School of Medicine and Dental Sciences, Asahimachi-Dori 1-754, Niigata 951-8520, Japan.

e-mail: chenjun@med.niigata-u.ac.jp 
important to identify the risk factors of esophageal adenocarcinoma, as such understanding could help reduce the incidence of this disease if the risk factors include controllable lifestyle factors such as tobacco and alcohol use.

We evaluated the effects of tobacco smoking, alcohol consumption, diet habit, and family history of cancer on the incidence of esophageal adenocarcinoma. In particular, we attempted to estimate the interactive correlation with the amount and duration of alcohol and tobacco use. For this purpose, matched pair data consisting of a group of patients with esophageal adenocarcinoma and their lifestyle factors were collected using data from a case-control study conducted in Hebei Province, China.

\section{Subjects and Methods}

\section{Study design}

In this matched case-control study, 98 patients with ages below 65 years who had been diagnosed with esophageal adenocarcinoma and had initial surgery (cases) were matched with 294 healthy adults (controls). Using the software $\mathrm{R}$ packages, the cases were matched with controls at a ratio of 1:3 according to gender and age. Eligible subjects were adults living in the city of Shijiazhuang, Hebei Province, China. Before the interview survey, the subjects were informed of all study objectives and the content of this research. The research protocol was approved by the local ethics committee and the research committee of Hebei Medical University.

\section{Subjects}

The cases were 98 patients who underwent initial resection for esophageal adenocarcinoma between January 2002 and December 2006 at the Fourth Hospital of Hebei Medical University in Shijiazhuang, Hebei Province. All patients were diagnosed by histological examination. Using a physical examination database, 3076 healthy persons who lived in Hebei Province from 2002 to 2006 were enrolled as controls and underwent medical checkups.

\section{Risk factors}

The factors and categories used in the study are shown in Tables 1 and 2. Data for subjects were obtained from hospital medical records (for cases) or from the check-up forms from physical examinations (for controls). Information on alcohol consumption consisted of average grams of alcohol consumed per day, type of alcoholic beverage consumed, lifetime duration of alcohol use, ages when alcohol consumption began and quitted, and number of years since quitting. The type of beverage consumed was classified as baijiu, beer, and other liquors. Baijiu is a Chinese distilled alcoholic beverage usually made from sorghum or glutinous rice. It is the most popular liquor in China and contains $40 \%$ to $60 \%$ alcohol by volume on average. Chinese usually drink baijiu in a single swallow from a 20 to $30 \mathrm{ml}$ glass, without a chaser. The average daily amount of alcohol was calculated by using alcohol exchange rates of $40 \%$ for baijiu, $5 \%$ for beer, $12 \%$ for wine, and $4 \%$ for hard liquor. One drink was defined as $50 \mathrm{ml}$ of baijiu, $400 \mathrm{ml}$ of beer, $167 \mathrm{ml}$ of wine, or $50 \mathrm{ml}$ of hard liquor. A nondrinker was defined as someone who consumed less than 1 drink per month. The total amount of pure alcohol consumed per day was calculated using the abovementioned alcohol percentages.

Finally, the risk factors used in this study were summarized with respect to the amount of alcohol per day, the duration of alcohol consumption (years), the interaction between the amount and duration of alcohol consumption; the number of cigarettes per day, the duration of tobacco smoking (years), the interaction between the amount and duration of tobacco smoking; the diet habit and family history of cancer. The interaction between the amount and duration of alcohol consumption for 20 grams of alcohol per day for 10 years and that for 10 grams of alcohol per day for 20 years were distinguished by slightly modifying partial dummy variables, as described in the next section. In other words, their interactions represent the patterns or habits of drinking consumption and smoking.

\section{Statistical analysis}

We performed univariate and multivariate conditional logistic regression analyses to estimate odds ratios (ORs) and the corresponding $95 \%$ confidence intervals (CIs). ORs represent the increased odds of incident esophageal adenocarcinoma among cases as compared with controls. The candidate factors were amount of alcohol consumption, duration of alcohol consumption, interaction between the amount and duration of alcohol consumption, amount of tobacco smoked, duration of tobacco smoking, interaction between the amount and duration of tobacco smoking, diet habit, and family history of cancer. Amount and duration were divided into 4 categories. The cut-off thresholds of categorical variables were determined by common usage informed by interview before analysis. These variables were then converted into the dummy variables with 3 categories. The reference categories were defined as nonsmokers and/or nondrinkers. For the interaction between the amount and duration of alcohol consumption, the dummy variable was created so that individuals drinking 10 milliliters per day for 20 years could be discriminated from those drinking 20 milliliters per day for 10 years, even though the total amounts consumed are identical. The diet habit was categorized as hot, fast, hot plus fast and roughage food four kinds'habits compare to normal; and family history of cancer was separated as had or hadn't by yes and no. All statistical analyses were performed using SAS 9.1. A $P$ value less than 0.05 was considered statistically significant for all tests.

\section{Results}

\section{Subject characteristics}

The life expectancy of the study area was around 70 years old. To avoid interference from the presence of numerous factors related to age, we selected cases and controls that were younger than 65 years. The demographic characteristics of cases and controls are shown in Table 1. There were 34 cases and 102 controls younger than 54 years, and 64 cases and 192 controls 55 to 64 years of age. As anticipated, there were higher proportions of non-drinkers and non-smokers among controls. However, the proportions of individuals with a normal diet habit and a family history of cancer were almost equal in cases and controls. Among regular drinkers, there was a higher proportion of heavy drinking and higher proportions for each duration of alcohol use in cases as compared with controls. Among regular smokers, there were slightly higher proportions of cases for each amount and duration of tobacco use.

Table 2 shows the detailed distributions of alcohol and tobacco use. Among subjects consuming more than $30 \mathrm{ml}$ 
Table 1. Baseline characteristics of controls and cases.

\begin{tabular}{|c|c|c|c|c|}
\hline \multirow{2}{*}{ Factors } & \multirow{2}{*}{ Categories } & \multicolumn{3}{|c|}{ No. of subjects (\%) } \\
\hline & & Controls $(n=294)$ & Cases $(n=98)$ & Total $(n=392)$ \\
\hline \multirow[t]{2}{*}{ Age (years) ${ }^{\mathrm{a}}$} & $<54$ & $102(34.7)$ & $34(34.7)$ & $136(34.7)$ \\
\hline & $55-64$ & $192(65.3)$ & $64(65.3)$ & $256(65.3)$ \\
\hline \multirow[t]{2}{*}{ Sex } & Male & $219(74.5)$ & $73(74.5)$ & $292(74.5)$ \\
\hline & Female & $75(25.5)$ & $25(25.5)$ & $100(25.5)$ \\
\hline \multirow[t]{4}{*}{ Amount of alcohol intake (g/day) } & 0 & $232(78.8)$ & $51(52.0)$ & $283(72.2)$ \\
\hline & $<15$ & $19(6.5)$ & $6(6.1)$ & $25(6.4)$ \\
\hline & $15-30$ & $19(6.5)$ & $7(7.1)$ & $26(6.6)$ \\
\hline & $>30$ & $24(8.2)$ & $34(34.8)$ & $58(14.8)$ \\
\hline \multirow{4}{*}{$\begin{array}{l}\text { Duration of alcohol consumption } \\
\text { (years) }\end{array}$} & 0 & $232(78.8)$ & $51(52.0)$ & $283(72.2)$ \\
\hline & $<16$ & $26(8.8)$ & $13(13.3)$ & $39(9.9)$ \\
\hline & $16-25$ & $23(7.8)$ & $17(17.3)$ & $40(10.2)$ \\
\hline & $>25$ & $13(4.4)$ & $17(17.4)$ & $30(7.7)$ \\
\hline \multirow{4}{*}{$\begin{array}{l}\text { Amount of tobacco smoking } \\
\text { (cig/day) }\end{array}$} & 0 & $166(56.5)$ & $39(39.8)$ & $205(52.3)$ \\
\hline & $<11$ & $41(13.9)$ & $18(18.4)$ & $59(15.1)$ \\
\hline & $11-20$ & $59(20.1)$ & $30(30.6)$ & $89(22.7)$ \\
\hline & $>20$ & $28(9.5)$ & $11(11.2)$ & $39(9.9)$ \\
\hline \multirow{4}{*}{$\begin{array}{l}\text { Duration of tobacco smoking } \\
\text { (years) }\end{array}$} & 0 & $166(56.5)$ & $39(39.8)$ & $205(52.3)$ \\
\hline & $<21$ & $62(21.1)$ & $22(22.4)$ & $84(21.4)$ \\
\hline & $21-30$ & $50(17.0)$ & $28(28.6)$ & $78(19.9)$ \\
\hline & $>30$ & $16(5.4)$ & $9(9.2)$ & $25(6.4)$ \\
\hline \multirow[t]{5}{*}{ Diet habits } & Normal & $196(66.7)$ & $64(65.3)$ & $260(66.3)$ \\
\hline & Hot food & $36(12.2)$ & $9(9.2)$ & $45(11.5)$ \\
\hline & Fast food & $29(9.9)$ & $10(10.2)$ & $39(9.9)$ \\
\hline & Hot + fast food & $25(8.5)$ & $13(13.3)$ & $38(9.7)$ \\
\hline & Roughage food & $8(2.7)$ & $2(2.0)$ & $10(2.6)$ \\
\hline \multirow[t]{2}{*}{ Family history of cancer } & No & $254(86.4)$ & $78(79.6)$ & $332(84.7)$ \\
\hline & Yes & $40(13.6)$ & $20(20.4)$ & $60(15.3)$ \\
\hline
\end{tabular}

${ }^{a}$ Age refers to age at diagnosis for cases and age at time of medical checkup for controls.

of alcohol per day, there were higher proportions of cases than controls for all durations of alcohol consumption.

\section{Estimation of the risk for esophageal adenocarcinoma by univariate conditional logistic regression analysis}

Conditional logistic regression analysis was performed to estimate the risk for incident esophageal adenocarcinoma. Table 3 shows the OR and corresponding 95\% CI for each candidate risk factor. The ORs for the durations of alcohol consumption and tobacco smoking tended to monotonically increase with increasing duration. Regarding duration of alcohol consumption, the estimated ORs for $<$ 16 years, 16 to 25 years, and $>25$ years were $3.11,4.44$, and 7.61, respectively. Regarding duration of tobacco smoking, the ORs for $<21$ years, 21 to 30 years, and $>30$ years were $2.48,3.89$, and 3.65 , respectively.

However, the ORs for amount of alcohol consumed and amount of tobacco smoked were not always monotonic. With respect to the former, the ORs for $<15,15$ to 30 , and $>30 \mathrm{ml} /$ day were estimated at $2.79,1.87$, and 10.58 , respectively. The OR for consumption of $>30 \mathrm{ml} /$ day was markedly higher $(P<0.001)$. In contrast, the ORs for amount of tobacco smoked ranged from approximately 2 to 4. Diet habit and family history of cancer were not associated with esophageal adenocarcinoma. These factors were thus removed from subsequent analysis.

Interaction between alcohol consumption and tobacco smoking and the risk of esophageal adenocarcinoma

Three variables - alcohol consumption, tobacco smoking, and their interaction - were used to calculate estimated coefficients, standard errors, and $P$ values by means of conditional logistic regression analysis of the risk of esophageal adenocarcinoma (Table 4). On multivariate analysis, the estimated regression coefficients for alcohol consumption, tobacco smoking, and their interaction were $1.28,0.72$, and $0.07(P=0.04,0.11$, and 0.92$)$, respectively, which indicates that alcohol consumption is a significant risk factor for esophageal adenocarcinoma and that tobacco smoking had no synergistic effect on esophageal adenocar- 
Table 2. Contingency table for amount and duration of alcohol consumption (a) and tobacco smoking (b).

(a) Distribution of alcohol consumption.

\begin{tabular}{|c|c|c|c|c|c|c|}
\hline \multirow{3}{*}{ Duration (years) } & \multicolumn{6}{|c|}{ No. of subjects (\%) } \\
\hline & \multicolumn{2}{|c|}{$<15 \mathrm{ml} /$ day } & \multicolumn{2}{|c|}{$15-30 \mathrm{ml} /$ day } & \multicolumn{2}{|c|}{$>30 \mathrm{ml} /$ day } \\
\hline & Controls & Cases & Controls & Cases & Controls & Cases \\
\hline$<16$ & $9(3.1)$ & $3(3.1)$ & $8(2.7)$ & $4(4.1)$ & $9(3.1)$ & $6(6.1)$ \\
\hline $16-25$ & $4(1.4)$ & $2(2.0)$ & $8(2.7)$ & $2(2.0)$ & $11(3.7)$ & $13(13.3)$ \\
\hline$>25$ & $6(2.0)$ & $1(1.0)$ & $3(1.0)$ & $1(1.0)$ & $4(1.4)$ & $15(15.3)$ \\
\hline
\end{tabular}

47 cases and 62 controls were classified as drinkers; the above percentages were calculated based on denominators of 294 and 98 for controls and cases, respectively.

(b) Distribution of tobacco smoking.

\begin{tabular}{|c|c|c|c|c|c|c|}
\hline \multirow{3}{*}{ Duration (years) } & \multicolumn{6}{|c|}{ No. of subjects (\%) } \\
\hline & \multicolumn{2}{|c|}{$<11 \mathrm{cig} /$ day } & \multicolumn{2}{|c|}{$11-20 \mathrm{cig} /$ day } & \multicolumn{2}{|c|}{$>20 \mathrm{cig} /$ day } \\
\hline & Controls & Cases & Controls & Cases & Controls & Cases \\
\hline$<21$ & $19(6.5)$ & $8(8.2)$ & $29(9.9)$ & $11(11.2)$ & $14(4.8)$ & $3(3.1)$ \\
\hline $21-30$ & $18(6.1)$ & $9(9.2)$ & $19(6.5)$ & $12(12.3)$ & $13(4.4)$ & $7(7.1)$ \\
\hline$>30$ & $4(1.4)$ & $1(1.0)$ & $11(3.7)$ & $7(7.1)$ & $1(0.3)$ & $1(1.0)$ \\
\hline
\end{tabular}

59 cases and 128 controls were classified as smokers; the above percentages are calculated based on denominators of 294 and 98 for controls and cases, respectively.

cinoma incidence.

Risk of esophageal adenocarcinoma with respect to the combination of the amount and duration of alcohol consumption

Univariate conditional logistic regression analysis was used to estimate ORs for categories that combined the amount and duration of alcohol consumption (Table 5). The analysis revealed that, as compared with non-drinkers, consumption of $>30 \mathrm{ml} /$ day of alcohol was associated with significantly higher ORs (< 16 yr: OR, 5.45; $16-25$ yr: OR, 9.07; > 25 yr: OR, 24.56), irrespective of the duration of alcohol consumption. However, the ORs of the groups with the lowest daily alcohol consumption were always less than 5 , and the lowest OR for esophageal adenocarcinoma among drinkers was 1.24 .

\section{Discussion}

Primary adenocarcinoma of the esophagus was uncommon in the past, representing only 10 percent of all esophageal cancers. However, during the past decade there has been a striking increase in incidence. Although the cause of this dramatic increase is unclear, recent studies have shown a marked similarity in age distribution, sex ratio, race, clinical presentation, and pathologic features between adenocarcinoma in Barrett's esophagus and adenocarcinoma of the cardia, which suggests that there are common etiologic factors between these two conditions, such as gastroesophageal reflux and increased alcohol and tobacco consumption (Brown and Devesa 2002; Post et al. 2007; Brown et al.
2008). This prompted us to conduct the current study, which sought to clarify the adverse effects of alcohol and tobacco useage on the development of esophageal adenocarcinoma. Our findings clearly demonstrated that alcohol consumption is an independent risk factor for esophageal adenocarcinoma, after adjusting for the effect of tobacco smoking, and that heavy daily alcohol consumption dramatically increased the risk for esophageal adenocarcinoma, independent of the duration.

In a previous study, we found that tobacco and alcohol use were the dominant risk factors for esophageal squamous cell carcinoma in this region of China (Chen et al. 2010), whereas the present findings revealed that the dominant risk factor for esophageal adenocarcinoma was heavy alcohol consumption, and that tobacco smoking was only a weak risk factor. On univariate analysis, the highest ORs with respect to the duration of use were 7.61 for alcohol consumption longer than 25 years and 3.89 for tobacco smoking for 21 to 30 years. The highest ORs with respect to amount used per day were 10.58 for $>30 \mathrm{ml}$ of alcohol per day and 3.64 for smoking 11 to 20 cigarettes per day. In both cases, the ORs for alcohol use were considerably higher than those associated with tobacco use.

The interaction between tobacco smoking and alcohol drinking has been extensively studied, and it has been suggested that they interact in a multiplicative manner with respect to the risk of esophageal cancer (De Stefani et al. 1990; Lee et al. 2007; Brown et al. 2008). However, Steevens et al. (2010) found no interaction effect in their prospective study of the risk of adenocarcinoma. Their 
Table 3. Odds ratios and $95 \%$ confidence intervals for various risk factors on univariate conditional logistic regression analysis.

\begin{tabular}{|c|c|c|c|}
\hline Factors & Odds Ratio & 95\% Confidence Interval & $P$ value \\
\hline \multicolumn{4}{|c|}{ Amount of alcohol intake (ml/day) } \\
\hline 0 & 1.00 & & \\
\hline$<15$ & 2.79 & $0.93-8.41$ & 0.07 \\
\hline $15-30$ & 1.87 & $0.73-4.81$ & 0.19 \\
\hline$>30$ & 10.58 & $4.73-23.68$ & $<0.001$ \\
\hline \multicolumn{4}{|c|}{ Duration of alcohol consumption (years) } \\
\hline 0 & 1.00 & & \\
\hline$<16$ & 3.11 & $1.35-7.16$ & 0.007 \\
\hline $16-25$ & 4.44 & $2.02-9.76$ & $<0.001$ \\
\hline$>25$ & 7.61 & $3.19-18.17$ & $<0.001$ \\
\hline \multicolumn{4}{|c|}{ Amount of tobacco smoking (cig/day) } \\
\hline 0 & 1.00 & & \\
\hline$<11$ & 3.02 & $1.33-6.87$ & 0.008 \\
\hline $11-20$ & 3.64 & $1.72-7.71$ & $<0.001$ \\
\hline$>20$ & 2.76 & $1.11-6.91$ & 0.03 \\
\hline \multicolumn{4}{|c|}{ Duration of tobacco smoking (years) } \\
\hline 0 & 1.00 & & \\
\hline$<21$ & 2.48 & $1.13-5.47$ & 0.02 \\
\hline $21-30$ & 3.89 & $1.81-8.36$ & $<0.001$ \\
\hline$>30$ & 3.65 & $1.32-10.08$ & 0.01 \\
\hline \multicolumn{4}{|l|}{ Diet habits } \\
\hline Normal diet & 1.00 & & \\
\hline Hot food & 0.79 & $0.37-1.71$ & 0.55 \\
\hline Fast food & 1.09 & $0.49-2.43$ & 0.83 \\
\hline Hot + fast food & 1.65 & $0.76-3.57$ & 0.20 \\
\hline Roughage food & 0.78 & $0.16-3.92$ & 0.76 \\
\hline \multicolumn{4}{|c|}{ Family history of cancer } \\
\hline No & 1.00 & & \\
\hline Yes & 1.64 & $0.90-3.00$ & 0.11 \\
\hline
\end{tabular}

Table 4. Result of multivariate conditional logistic regression analysis of the risk of esophageal adenocarcinoma including three factors of alcohol consumption, tobacco smoking and the interaction between alcohol consumption and tobacco smoking.

\begin{tabular}{lccc}
\hline \multicolumn{1}{c}{ Factors } & Estimated coefficient & Standard error & $P$ value \\
\hline Alcohol consumption & 1.28 & 0.64 & 0.04 \\
Tobacco smoking & 0.72 & 0.45 & 0.11 \\
Interaction of alcohol and tobacco use & 0.07 & 0.70 & 0.92 \\
\hline
\end{tabular}

results and those of the present study indicate that the joint effect of smoking and alcohol might not be a significant factor in all populations. On conditional logistic regression analysis, the estimated coefficient of interaction between tobacco smoking and alcohol drinking (0.07) was lower than those for alcohol drinking (1.28) and tobacco smoking $(0.72)$ alone. The interaction of smoking and drinking in adenocarcinoma is different to what was observed in squamous cell carcinoma, which is mainly esophageal cancer.

Using conditional logistic regression analysis, we found that the interaction between the amount and duration of alcohol consumption (i.e., average daily alcohol intake multiplied by duration of intake) was the most significant risk factor in the incidence of esophageal adenocarcinoma. This finding is of particular interest because it indicates that the interaction between the amount and duration of alcohol consumption has more predictive power than either of these two factors considered individually. As shown in Table 5, heavy drinkers had the highest risk for esophageal adenocarcinoma, particularly those who consumed more than 30 milliliters daily. The ORs for heavy drinkers were 5 to 24 times of those of non-drinkers. 
Table 5. Odds ratios and $95 \%$ confidence intervals for combinations of amount and duration of alcohol consumption on univariate conditional logistic regression analysis.

\begin{tabular}{cccc}
\hline Factors & Odds Ratio & $95 \%$ Confidence Interval & $P$ value \\
\hline $\begin{array}{c}\text { Amount and duration of alcohol use } \\
<15 \mathrm{ml} \text { and }<16 \mathrm{yr}\end{array}$ & 2.70 & & \\
$<15 \mathrm{ml}$ and $16-25 \mathrm{yr}$ & 4.85 & $0.61-11.90$ & 0.19 \\
$<15 \mathrm{ml}$ and $>25 \mathrm{yr}$ & 1.60 & $0.17-30.15$ & 0.09 \\
$15-30 \mathrm{ml}$ and $<16 \mathrm{yr}$ & 3.31 & $0.87-12.62$ & 0.68 \\
$15-30 \mathrm{ml}$ and $16-25 \mathrm{yr}$ & 1.24 & $0.25-6.23$ & 0.08 \\
$15-30 \mathrm{ml}$ and $>25 \mathrm{yr}$ & 1.28 & $0.13-12.88$ & 0.79 \\
$>30 \mathrm{ml}$ and $<16 \mathrm{yr}$ & 5.45 & $1.51-19.75$ & 0.83 \\
$>30 \mathrm{ml}$ and $16-25 \mathrm{yr}$ & 9.07 & $3.24-25.36$ & 0.01 \\
$>30 \mathrm{ml}$ and $>25 \mathrm{yr}$ & 24.56 & $7.11-84.78$ & $<0.001$ \\
\hline
\end{tabular}

The strength of our study was its use of a case-control design to estimate the effects of the amount and duration of alcohol and tobacco use separately and in combination. However, this study did have limitations that warrant discussion. In China, the main type of esophageal cancer is squamous cell carcinoma. Because of this, the sample size was smaller than expected, which could result in bias. However, to address this, we used 1:3 matching to increase accuracy. Due to the life expectancy of study area was around 70 years old and people older than 65 are subject to complications of multiple diseases, only those people younger than 65 years old were selected for the study. As a local guidance of therapeutic principle, the first choice of treatment to the esophageal cancer patients was surgery operation. So the cases were selected from postoperated patients and excluded nonoperable esophageal cancer patients. We estimated the risks for esophageal adenocarcinoma using information on alcohol, smoking, family history of cancer, and diet; we did not collect data on other possible risk factors, e.g., social status, because we were unable to obtain precise data on income and diet in healthy people in Hebei Province, particularly among agricultural workers. And also some other potential risk factors, such as gastroesophageal reflux disease, and body mass index, were not included in due to the lower prevalence or imperfect of the data source (Ho et al. 1998; Dent et al. 2005; Yu 2011). Finally, because only a very small number of people (less than three percent in each group) successfully quit drinking or smoking in this study, we could not include cessation of alcohol or tobacco use in the analysis. Nevertheless, assuming our findings reflect true causal associations, it seems prudent to advise heavy drinkers to undergo more frequent physical examinations, which might result in earlier diagnosis of this cancer.

\section{Conclusions}

Our results confirm that alcohol consumption, especially heavy alcohol use, increases the risk of esophageal adenocarcinoma. From a public health perspective, our findings indicate that heavy alcohol users are at a particu- larly high risk of esophageal adenocarcinoma, especially the $14.8 \%$ of this population who consume more than $30 \mathrm{ml}$ of alcohol per day. We expect that future studies will identify more sensitive risk factors for esophageal adenocarcinoma.

\section{Acknowledgments}

We would like to thank the patients who accepted the survey in this study.

\section{Conflict of Interest}

The authors declare no conflict of interest.

\section{References}

Blot, W.J., Devesa, S.S., Kneller, R.W. \& Fraumeni, J.F. Jr. (1991) Rising incidence of adenocarcinoma of the esophagus and gastric cardia. JAMA, 265, 1287-1289.

Bollschweiler, E., Wolfgarten, E., Gutschow, C. \& Hölscher, A.H. (2001) Demographic variations in the rising incidence of esophageal adenocarcinoma in white males. Cancer, 92, 549555.

Botterweck, A.A., Schouten, L.J., Volovics, A., Dorant, E. \& van Den Brandt, P.A. (2000) Trends in incidence of adenocarcinoma of the oesophagus and gastric cardia in ten European countries. Int. J. Epidemiol. 29, 645-654.

Brown, L.M. \& Devesa, S.S. (2002) Epidemiologic trends in esophageal and gastric cancer in the United States. Surg. Oncol. Clin. N. Am., 11, 235-256.

Brown, L.M., Devesa, S.S. \& Chow, W.H. (2008) Incidence of adenocarcinoma of the esophagus among white Americans by sex, stage, and age. J. Natl. Cancer Inst., 100, 1184-1187.

Brown, L.M., Hoover, R., Silverman, D., Baris, D., Hayes, R., Swanson, G.M., Schoenberg, J., Greenberg, R., Liff, J., Schwartz, A., Dosemeci, M., Pottern, L. \& Fraumeni, J.F. Jr. (2001) Excess incidence of squamous cell esophageal cancer among US Black men: role of social class and other risk factors. Am. J. Epidemiol., 153, 114-122.

Chen, J., Zhang, N., Wakai, T., Wei, L., He, Y., Kumagai, N., Kitsu, K., Wang, S. \& Akazawa, K. (2010) Effect of the interaction between the amount and duration of alcohol consumption and tobacco smoking on the risk of esophageal cancer: a casecontrol study. Experimental and Therapeutic Medicine, 152, 991-997.

Chen, M.Y., Ott, D.J. \& Gelfand, D.W. (1995) More evidence for the increasing prevalence of adenocarcinoma of the esophagus over an 18-year period. J. Clin. Gastroenterol, 21, 254-255. 
Crane, L.M., Schaapveld, M., Visser, O., Louwman, M.W., Plukker, J.T. \& van Dam, G.M. (2007) Oesophageal cancer in the Netherlands: increasing incidence and mortality but improving survival. Eur. J. Cancer, 43, 1445-1451.

Dent, J., El - Serag, H.B., Wallander, M.A. \& Johansson, S. (2005) Epidemiology of gastroesophageal reflux disease: a systematic review. Gut, 54, 710-717.

De Stefani, E., Muñoz, N., Estève, J., Vasallo, A., Victora, C.G. \& Teuchmann, S. (1990) Mate drinking, alcohol, tobacco, diet, and esophageal cancer in Uruguay. Cancer Res., 50, 426-431.

Devesa, S.S., Blot, W.J. \& Fraumeni, J.F. Jr. (1998) Changing patterns in the incidence of esophageal and gastric carcinoma in the United States. Cancer, 83, 2049-2053.

Engel, L.S., Chow, W.H., Vaughan, T.L., Gammon, M.D., Risch, H.A., Stanford, J.L., Schoenberg, J.B., Mayne, S.T., Dubrow, R., Rotterdam, H., West, A.B., Blaser, M., Blot, W.J., Gail, M.H. \& Fraumeni, J.F. Jr. (2003) Population attributable risks of esophageal and gastric cancers. J. Natl. Cancer Inst., 95, 1404-1413.

Fan, Y.J., Song, X., Li, J.L., Li, X.M., Liu, B., Wang, R., Fan, Z.M. \& Wang, L.D. (2008) Esophageal and gastric cardia cancers on 4238 Chinese patients residing in municipal and rural regions: a histopathological comparison during 24-year period. World J. Surg., 32, 1980-1988.

Freedman, N.D., Park, Y., Subar, A.F., Hollenbeck, A.R., Leitzmann, M.F., Schatzkin, A. \& Abnet, C.C. (2007) Fruit and vegetable intake and esophageal cancer in a large prospective cohort study. Int. J. Cancer, 121, 2753-2760.

Ho, K.Y., Kang, J.Y. \& Seow, A. (1998) Prevalence of gastrointestinal symptoms in a multiracial Asian population, with particular reference to reflux-type symptoms. Am. J. Gastroenterol., 93, 1816-1822.

Lee, C.H., Wu, D.C., Lee, J.M., Wu, I.C., Goan, Y.G., Kao, E.L., Huang, H.L., Chan, T.F., Chou, S.H., Chou, Y.P., Lee, C.Y., Chen, P.S., Ho, C.K., He, J. \& Wu, M.T. (2007) Carcinogenetic impact of alcohol intake on squamous cell carcinoma risk of the oesophagus in relation to tobacco smoking. Eur. J. Cancer, 43, 1188-1199.

Pera, M., Cameron, A.J., Trastek, V.F., Carpenter, H.A. \& Zinsmeister, A.R. (1993) Increasing incidence of adenocarcinoma of the esophagus and esophagogastric junction. Gastroenterology, 104, 510-513.

Pohl, H. \& Welch, H.G. (2005) The role of overdiagnosis and reclassification in the marked increase of esophageal adenocarcinoma incidence. J. Natl. Cancer Inst., 97, 142-146.

Post, P.N., Siersema, P.D. \& Van Dekken, H. (2007) Rising incidence of clinically evident Barrett's oesophagus in The Netherlands: a nation-wide registry of pathology reports. Scand. J. Gastroenterol., 42, 17-22.

Steevens, J., Schouten, L.J., Goldbohm, R.A. \& van den Brandt, P.A. (2010) Alcohol consumption, cigarette smoking and risk of subtypes of oesophageal and gastric cancer: a prospective cohort study. Gut, 59, 39-48.

Wei, J.T. \& Shaheen, N. (2003) The changing epidemiology of esophageal adenocarcinoma. Semin. Gastrointest. Dis., 14, 112-127.

Yu, H.K. (2011) From GERD to Barrett's Esophagus: Is the Pattern in Asia Mirroring that in the West? J. Gastroenterol. Hepatol., Jan 25. 